\title{
Deterministic Model Investigation of Processes in a Heterogeneous E-Learning Environment
}

\author{
Radi Romansky, Technical University of Sofia, Bulgaria* \\ (iD) https://orcid.org/0000-0003-2228-9397 \\ Irina Noninska, Technical University of Sofia, Bulgaria
}

\begin{abstract}
The investigation of characteristics of access and use of resources in different distributed environments in the network space is aimed at determining optimal levels for the basic parameters of the supported processes. On the other hand, with the development of the possibilities of the digital space and the significant change in the level of informatization of the society, it is necessary to take the necessary measures to ensure secure access to information resources and in particular to the profiles of personal data. In this respect, the purpose of the article is to propose an organization of heterogeneous environment with resources stored in different places (own memories and cloud data centres). A general architecture and functionality of the main sub-systems are presented. Deterministic model investigation by using petri net apparatus based on preliminary formalization is provided to analyse the effectiveness of the processes for regulated and secure access to resources.
\end{abstract}

\section{KEYWORDS}

Access Regulation, Data Protection, Discrete Formalization, Functionality of E-Learning Environment, Heterogeneous Resources, Model Investigation

\section{INTRODUCTION}

The contemporary digital age is characterized by total informatization of society and expansion of the field of applicability of electronic services, which is discussed in a number of publications. As stated in (Chatterjee, 2021) "there has been an immense advancement of internet technology", analyzing the contribution of the Internet of Things (IoT) and its impact on human capital and people's daily lives. In addition, the article (Khouja et al, 2018) should be noted, where it is declared that information technology (IT) "is a very important aspect for higher education institutions in both teaching, research and administration" and the managers of these institutions realize their strategic importance. This determines the growing role of IT professionals in developing the right approach to managing IT and their successful implementation in educational institutions in accordance with their business strategies. In this regard, the article provides a systematic review of the literature using various scientific and unconventional data. In addition to the positive conclusions of the study, it should made point out some features that may adversely affect business objectives, such as the need for additional costs for equipment and licensing of products, creation of new structural units, training of employees, etc. 
The positive impact of modern information and communication technologies on management in higher education is also confirmed by the study in (Egoeze et al, 2018), which in conclusion confirms the need for the ever-expanding scope of their application in administrative services. The conclusions made in the article are based on questionnaires and the collected data are analyzed with the help of ANOVA.

Cloud computing (CC), a constantly evolving technology for providing services in the global network, also provides good opportunities for the realization and improvement of university education. A study of the factors influencing human capital and cloud perception is presented in (Hiran, 2021), and the results show that the importance of organizational factors is greater than that of technological, environmental and sociocultural factors. This should reflect on the approaches for the implementation of cloud computing in higher education institutions.

In the digital world, the use of new IT in all areas of public life, including university communications, requires proper organization of processes and ensuring secure access to distributed information resources and their legal use (Glet \& Kaczynski, 2020) based on the requirements for strict information security (Monev, 2020). The need for reliable regulation of these processes is growing by setting specific requirements for the protection of resources in access and use (Romansky \& Noniska, 2020a) and ensuring adequate cybersecurity in the network space. The result is the document of the European Commission "General Data Protect Regulation" in force since May 2018. The introduction of specific requirements such as "privacy by default" and "privacy by design" in organizing data processing environments, as well as the paradigm "right to be forgotten / to be erased " (replaced the previous one "right to be left alone") correspond to the inalienable rights of people for "right to privacy" and "right to data protection" (Romansky \& Noniska, 2020b).

As mentioned above, when developing a technological system, it is recommended to conduct a model study, through which certain quantitative estimates for basic functional attributes can be obtained (Digalovski \& Rafajlovski, 2020). On the basis of adequate initial formalization of the studied object and subsequent application of an appropriate modelling approach, useful conclusions can be made about the behaviour of the system and the development of the processes in it (Cheryshov et al., 2020). The success of this study depends on a properly defined experimental design based on predetermined sets of primary and secondary factors.

The aim of the article is to propose a new approach for common organization of a heterogeneous environment of information and training resources located in different places in the global network and using cloud computing and possibly social media. This poses serious challenges for IT professionals to provide platforms for the protection of information and personal data of human capital. Specifically, a general architectural concept of an e-learning system called Heterogeneous e-Learning Environment (HeLE) is proposed as a new concept. The study proposed here can be taken as a continuation of the problems discussed in (Romansky \& Noninska, 2016) and (Romansky \& Noninska, 2019). The contribution of the article can be determined by defining the functionality of the main subsystems, expressed in a set of procedures for regulating access to different resources (internal and external), the defined formalization of processes and proposed conceptual model, as well as in the organization of deterministic model investigation using the apparatus of a Petri net with discussion of the obtained analytical results. The goal of the article is to determine specific collection of procedures for counteracting the possible risk of illegitimate access and violation of the principles set by the "CIA triad" (confidentiality, integrity, availability) (Romansky \& Noniska, 2020a). The efficiency of the construction of the proposed environment is confirmed by an analysis of the behavior of the determined analytical model.

The article is organized as follows. The next section discusses some related work in the field. Section 3 presents the proposed generalized architectural model and functional sub-systems. The next two sections 4 and 5 are devoted to the presentation of the formalization and the conducted model investigation with analysis of the obtained results. Finally, conclusion and brief discussion is made in last section. 


\section{RELATED WORK}

It is known that the modelling is a suitable approach to determine the correctness of designed structures and processes in different areas. In essence, each mathematical model could be considered as a formalized descriptions of the object under investigation by using appropriate abstract language or mathematical formal system for describing functional behaviour, and computer tools and approaches are applied for all phase's realization. In this respect, it is necessary to specify that each research must be based on a preliminary defined conceptual model of the object under study. For example, a conceptual model with defined initial hypotheses was developed in (Chatterjee, 2021), which was applied in a deterministic model study and analysis of people's behavioral intentions to use IoT.

\section{Model Investigation of Computer Processes and Information Servicing}

There are different approaches to model investigation, but each of them is based on a certain descriptive formalism. One of these approaches is the analytical modelling and it is used in (Ding et al., 2020) for studying effect of thermal conductivity and geometric parameters on the thermal performance. It has been used for dimensionless design criterion to design the latent heat storage unit. Another example for application of mathematical modelling as an analytical approach is the study of biological systems presented in (Torres \& Santos, 2015). The goal of this article is to sum up the common procedures during the formalization and analysis of a biological problem from the perspective of Systems Biology, which approaches the study of biological systems as a whole, and the initial step is to define what a model of biological system is. The study is realised through three successive stages described in detail - formulation of a conceptual model, its mathematical formalization and analysis of the model results for optimization of the investigated system.

A review of analytical performance modeling for computer systems is presented in (Tay, 2020). This article states that each computer systems could be regarded as a combination of multiple hardware and software components and confirms the usefulness of applying analytical approaches for model investigation of system performance and efficiency of process. It discusses the motivation in constructing an analytical model in the field of computer engineering and presents some examples to show the suitability of model investigation with introducing some ideas. One useful conclusion of the article is that any computer system, which is a complex of interacting complex sub-systems, can be decomposed into separate relatively independent modules. This will allow their formalization and further analytical study, requiring the correct validation of the results. Appropriate validation of the presented concepts is made in (Antonelli et al., 2020) by applying analytical modeling for investigation of the efficiency of a cloud service organization, based on the formalization of the studied object as a queuing system with exponential stochastic input flows of requests and their service. The aim is to study the saturation limits of the system that uses the cloud infrastructure and service and to analyze its performance in the migration of virtual machines. The results of the model study have confirmed the adequate organization of the server service and the efficiency of the computational processes.

Although the information service processes are mostly stochastic in nature, the application of a deterministic approach to modelling has its advantages related to easier implementation, setup and verification. This is determined by the presence of well-developed on a theoretical and practical level systems that allow analytical functional approximation based on the correlation between ongoing processes. It is known that the deterministic approach can also be applied by replacing the probabilistic parameters of the studied system with their discrete statistical estimates (mean or final values). The application of such an approach is usually associated with the functional modeling of systems for studying the processes taking place in them, including multi-stage systems with an input flow of parallel tasks (Bolonkina et al., 2019). 


\section{Deterministic Modelling in the Digital World}

On the other hand, the behavior of different systems is characterized by asynchronous realization of events depending on the presence of certain conditions. This determines the applicability of the apparatus of Petri nets (PN) which permits to present the investigated process as a sequence of events (transitions) that can occur (realize) under certain conditions (positions). Various modifications of PN-theory are known, aiming at reflecting specific features of the studied objects - stochastic (Taleb-Berrouane et al., 2020), colored (Kai et al., 2020), fuzzy (Liu et al., 2020), etc. A new concept of extending PN-modelling celled "open" Petri net (OPN) is introduced in (Baez \& Master, 2020) which is defined as a set of connected PNs with glued outputs of one to the inputs of another. Such an OPN can be treated as a morphism of a symmetric monoidal system, described by two forms of semantics - operational semantics and accessibility semantics. Another example of the applicability of the Petri net apparatus is the study conducted in (Iakushkin, 2020), where a new approach called service-oriented Petri net (SOPN) is proposed for modeling interactions in service systems, which is an extension of the stochastic PN with additional components and capabilities.

Distributed information processing is a traditional feature of the contemporary digital society and is practically based on asynchronous processes in network space for resources management (Cheryshov et al., 2020). An example in this regard is the study of system performance and optimization criteria of service in multi-stage systems in planning tasks for execution based on heuristics presented in (Oleinikova et al., 2019). An analytical approach is proposed for the development of an algorithm using a set of heuristics, allowing at any time to decide which tasks to be performed. Distributed access to information resources is object of discussion in (Bayrakdar, 2020) aimed at managing communications in a wireless network of distributed independent sensors for monitoring objects. The proposed technique allows communications to be carried out by detecting inactive frequency bands and preventing interference.

Network security mechanisms are based on directory services, which in their developed version allow the user to create, modify, search, view and delete information. Basic features of the procedures for access control to corporate resources are discussed in (Cai et al., 2020), as a model for access control is proposed, preserving the known advantages of authorization with additional procedures for dynamic access control to the objects supported in the system. A continuation of the topic is made in (Christie et al., 2020) with a discussion of the requirements for establishing the identity of users and determining their permissions before accessing resources. The article focuses on the use of scientific gateways for this purpose and discusses scenarios for authentication and authorization of such third party identity platforms and services, offering architectural solutions.

In many cases, e-learning also uses the opportunities of social media for communication and knowledge sharing. This requires participants to be aware of network security and privacy issues, which is the subject of discussion in (Kanakaris et al, 2019). It is clearly stated that the use of social media has a potential negative impact with the possibility of violations, disclosure of available data, use of source code information, etc. This requires participants in these processes to be aware of the possible consequences and to take appropriate countermeasures, as well as poses an important task for IT professionals.

\section{FUNCTIONALITY OF E-LEARNING ENVIRONMENT}

\section{Generalized Architecture Model}

The main task of the contemporary technological society is to provide secure information services with reliable access to resources, which determines specific requirements for the infrastructure and organization of remote access. This also applies to modern e-learning technologies, which require new approaches and structures for administration and management of resources. The concept of active learning is established, in which the trained person exercises control over the learning process. 
Figure 1. Architecture of proposed HeLE

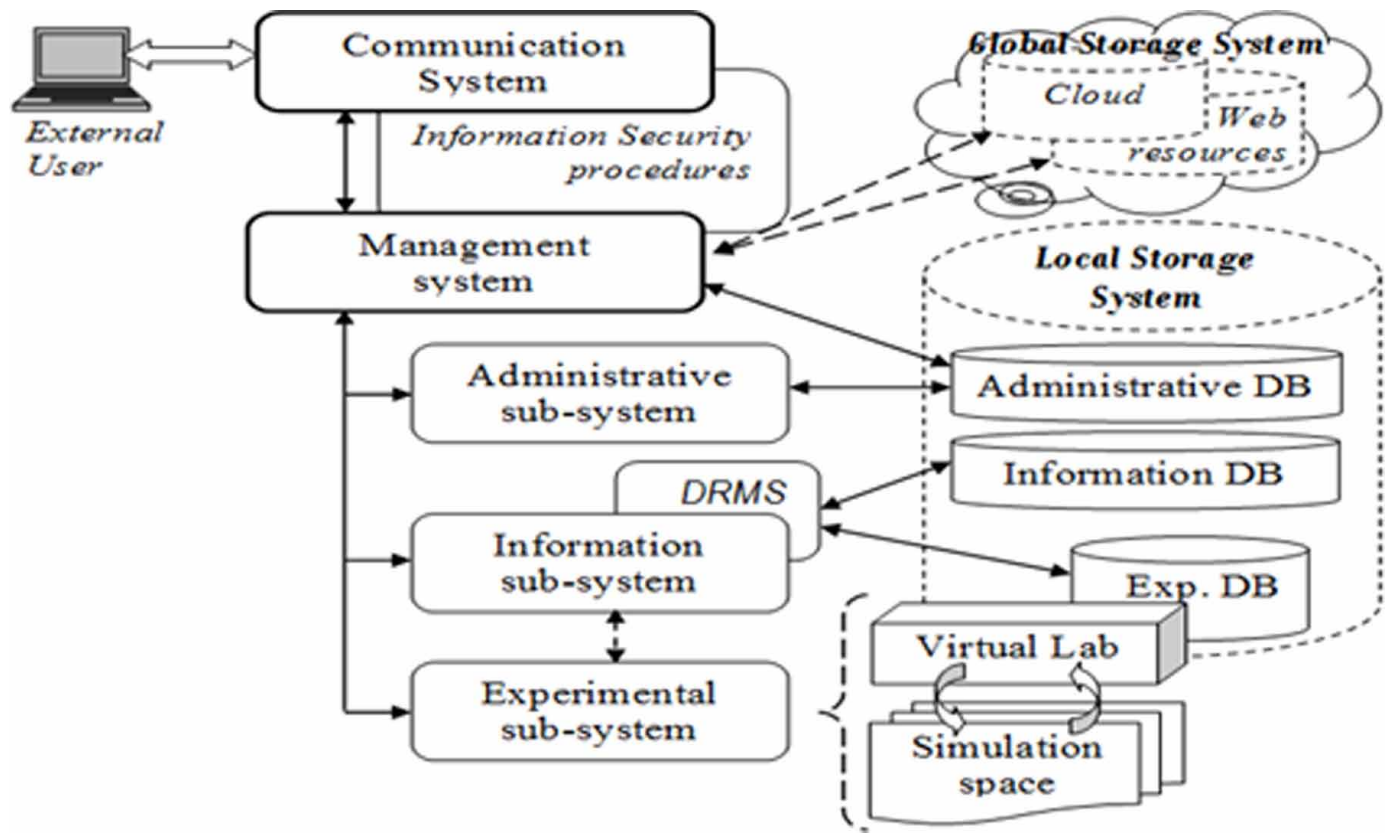

This requires constant access to information resources of various forms and contents, with cloud technologies offering an appropriate solution.

One proposal in this direction is the presented below distributed environment for active learning with information resources maintained and managed by different technologies, called Heterogeneous e-Learning Environment (HeLE). The goal is to formulate generalized organizational framework for regulated access to all supported heterogeneous resources (in cloud, in own storage, as a virtual spice, personal data, etc,) and ensuring secure and reliable use. An architectural model of HeLE for summarization of the main functionalities distributed between two basic systems is shown in Figure 1.

"Communication system (Front Office) to ensure reliable communication with users when processing requests and exchanging information objects. It is an entry point (portal) for remote access to the system of external users, which must provide reliable access control through identification, registration and verification. The implementation of these functionalities is supported by procedures for initial user registration and for identification and verification of access, and if successful, the request is transferred to the next (internal) level. Identification must ensure that the user can be identified. Upon registration, a new user profile is created, while only the data most necessary for this purpose is collected. Verification confirms the legitimacy of the user and access to system and information resources from the environment. An additional function is to maintain a log file for each access (time, IP address and other basic access attributes).

. Management system (Back Office) to ensure secure and reliable data processing and resource sharing when servicing an authenticated request. It is an internal system for administrative service taking into account the legitimacy of the requests and the level of granted rights for access to the requested resources. For the implementation of the planned functionalities it is necessary to maintain specialized procedures for request analysis, type of access, authentication, authorization, implementation of authorized access, maintenance of system and information resources, etc. To organize laboratory experiments, it is necessary to create and maintain a virtual environment for distributed simulations with the ability to evaluate activities and update work applications. 
Each of the two systems includes relatively independent sub-systems for the implementation of the planned functionalities for administration, organization and maintenance of information resources, providing an environment for laboratory tests, etc. Access to various resources is regulated by appropriate procedures, which are mainly implemented by DRMS (Digital Right Management System) - an important component of the management system.

\section{Functional Sub-systems}

The functions for ensuring information security are distributed between the two main systems, as the communication system realizes the initial level, while the main procedures are provided to the existing system. For this purpose, it provides several relatively independent sub-systems and objects.

- Administrative subsystem for complete administration of requests for access to resources with legitimacy verification and authorization (access rights). Its work is maintained by an administrative database, which stores all profiles of employees and users who have gone through initial registration. It is possible, through the functional assistance of the main management system, administrative data to be stored in data centres in cloud infrastructure.

- Information subsystem - integrates and manages the information fund of e-learning environment, providing secure and authorized access to information resources in the local storage and retrieval of context-sensitive information from him and through a management system for access to the global data storage (web-resources in the global network and cloud data centres). Its capabilities include access to and processing of data from experiments conducted with virtual tools for simulation research - Experimental Database (Exp. DB). The maintenance of the main functionalities when working with resources is performed by DRMS.

- Experimental subsystem - maintains a virtual laboratory with a suitable simulation space for conducting remote laboratory activities during training. For this purpose, the $3 \mathrm{D}$ functionalities of the virtual laboratory are used in the authorization of access and protection of resources. The subsystem provides reservation of time for work with laboratory equipment and control of the access mode, as well as management of remote experiments with systematization of experimental results, data storage and knowledge assessment.

- Virtual Lab for 3D simulations (Figure 2) - organization of experimental activity through virtual tools with the ability to obtain information on a selected topic from a local or global source with opportunities for discussions and mutual support of clients in the environment by maintaining synchronous (chat forums) and asynchronous (thematic forums) communications. When performing the functionalities, the 3D environment uses DRMS tools and host space for the work files

- Data storages and resources - sets of information and educational resources to support e-learning processes, distributed as internal (stored in their own memory) and external (stored in cloud data centres, including social media and networks, websites, etc.). To these are added administrative data and data and results of experiments.

\section{FORMALIZATION AND CONCEPTUAL MODEL}

When creating a conceptual model, a centralized "client-server" approach was adopted with a basic core web-based application, accessible via the Internet from various remote users. This provides an opportunity for multiple access to the information resources of the environment, distinguishing three user levels (Figure 2): 
Figure 2. Functional structure of Virtual Lab

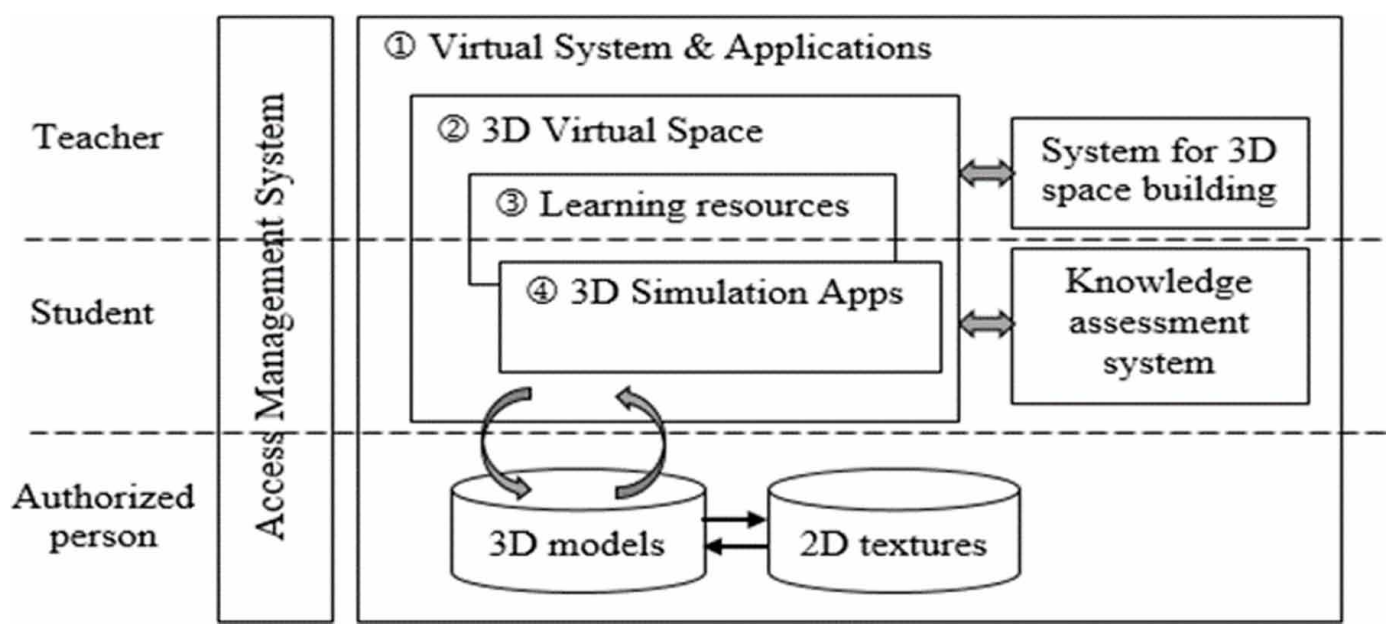

1. Teacher - there is an opportunity to interact with the web-based application for preparation of teaching material, realization of own virtual environment, creation of $3 \mathrm{D}$ simulations and organization of training through them.

2. Student-provides access to the information resources of the e-learning environment (embedded learning materials, 3D-models, simulation environment, etc.).

3. User (Authorized person) - has access to information about the functional capabilities of the learning environment, the way of organizing the learning processes and the composition of the environment for 3D-simulations, as well as opportunities for process administration.

The 3D environment is installed and executed on the server machine, and the visualization is performed on the client machines through a suitable browser and plug-in for presenting 3D content. The 3D models themselves run on remote server machines in the web space, and the common organization includes the following basic modules:

1. Web-based application - defines and implements different levels of access to the 3D learning environment. Provides a user interface for navigation and resource sharing. Responsible for the application of a specialized browser by the user, without which the $3 \mathrm{D}$ environment would not be visualized.

2. Three-dimensional environment-is realized with the help of software platforms for 3D modelling and it is built into an Internet-based application. The 3D models can be realized by using 3D Simulation Apps (see ,, in Figure 2).

3. 3Learning resources - determine the appropriate resources for displaying the learning content. They are introduced in a 3D environment through visualization methods in both the virtual 3D environment and the web-based application.

A chart presenting conceptual organization of processes and structures for protection of resources and services in the offered system is presented in Figures 3.

From the point of view of information processes, the environment allows the main participants in the procedures which are the basic human capital (administration, IT professionals, trainers/professors, learners/students and information resources) to be located in different geographically remote places. The learning processes are related to maintaining remote multi-user access to shared resources with 
Figure 3. Conceptual chart of the processes and structure of a protected system

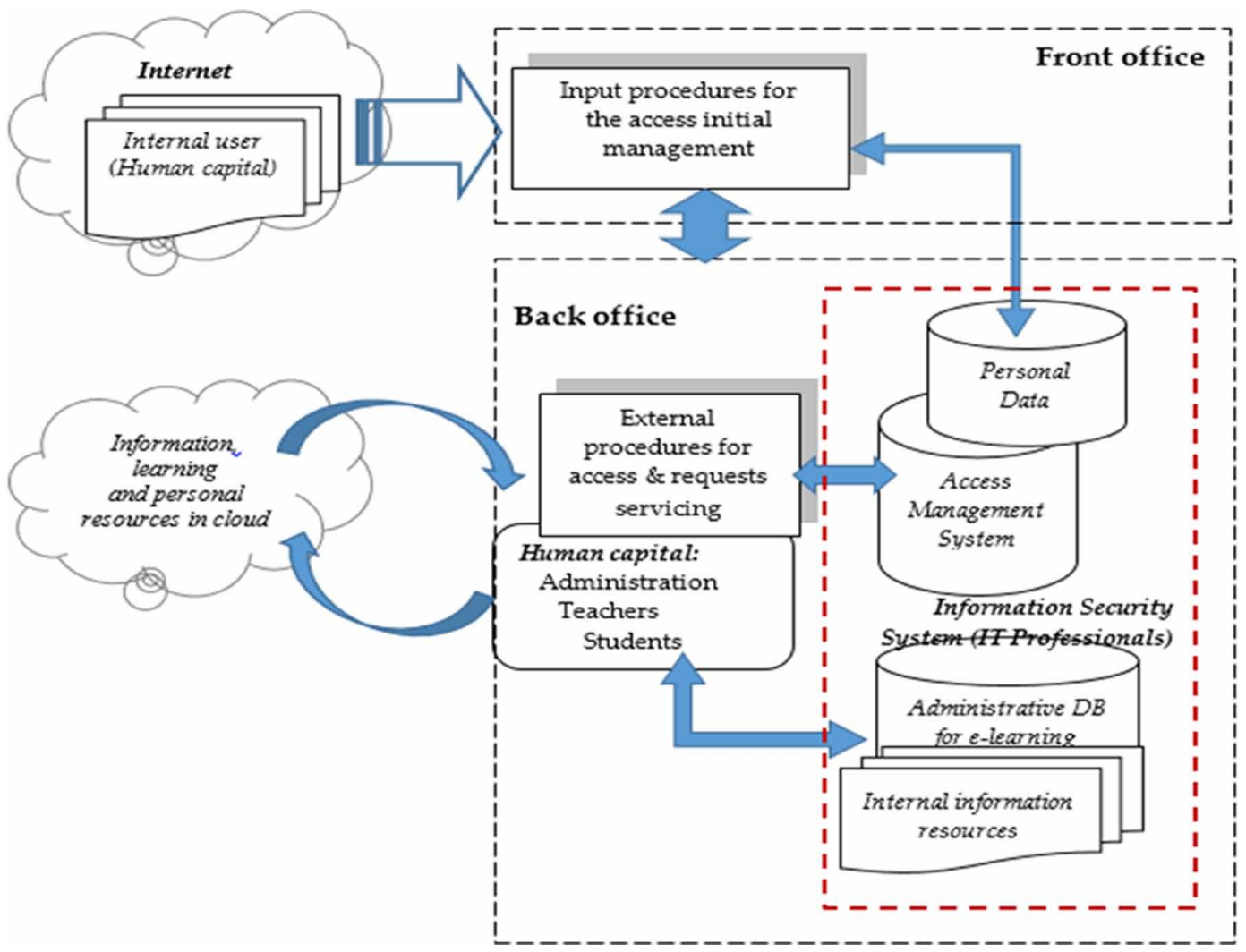

personalization for each client, which requires to define clear rules for secure access and using. This basic concept is reflected in the proposed conceptual model by defining two relatively independent sub-systems Front Office and Back Office), which identifie the need to establish clear levels of information security and protected access to all sensitive resources due to the different nature of users

For the purposes of a subsequent realization in real conditions, it is necessary to perform a model investigation, for which a mathematical formalization of the procedures and a description of the data flow must be performed. The formalization of the processes in the discussed system HeLS allows to differentiate the main procedures for regulating the access at different levels and providing reliable protection against errors and illegal changes of the resources, both from internal and external users. A graphical representation of the formalized environment in the form of an algorithmic description of the sequence of activities is shown in Figure 4. The functions of the communication and management systems (see Figure 1) are distributed between defined basic procedures - identification, registration, authentication, request analysis, authorization, and correct servicing.

To specify the sequence of processes and modes for access to the supported resources (personal data, information \& learning resources, system profiles, etc.), a Data Flow Diagram (DFD) is constructed, which is shown in Figure 5. DFD is an instrument to design a visual chart for presentation of the data flow between components of a developed system. The proposed diagram uses the elements form the notation of Chris Gane \& Trish Sarson (Li \& Chen, 2009), which are: (1) process (basic functional block); (2) data source or receiver (represents an input data flow generator or a data recipient); (3) stored data (form of structured data stored in memory as file, database, archive, information resource, training kit or another type); (4) directional arrows (to represent the movement 
Figure 4. Formalization of processes for access regulation in the HeLE

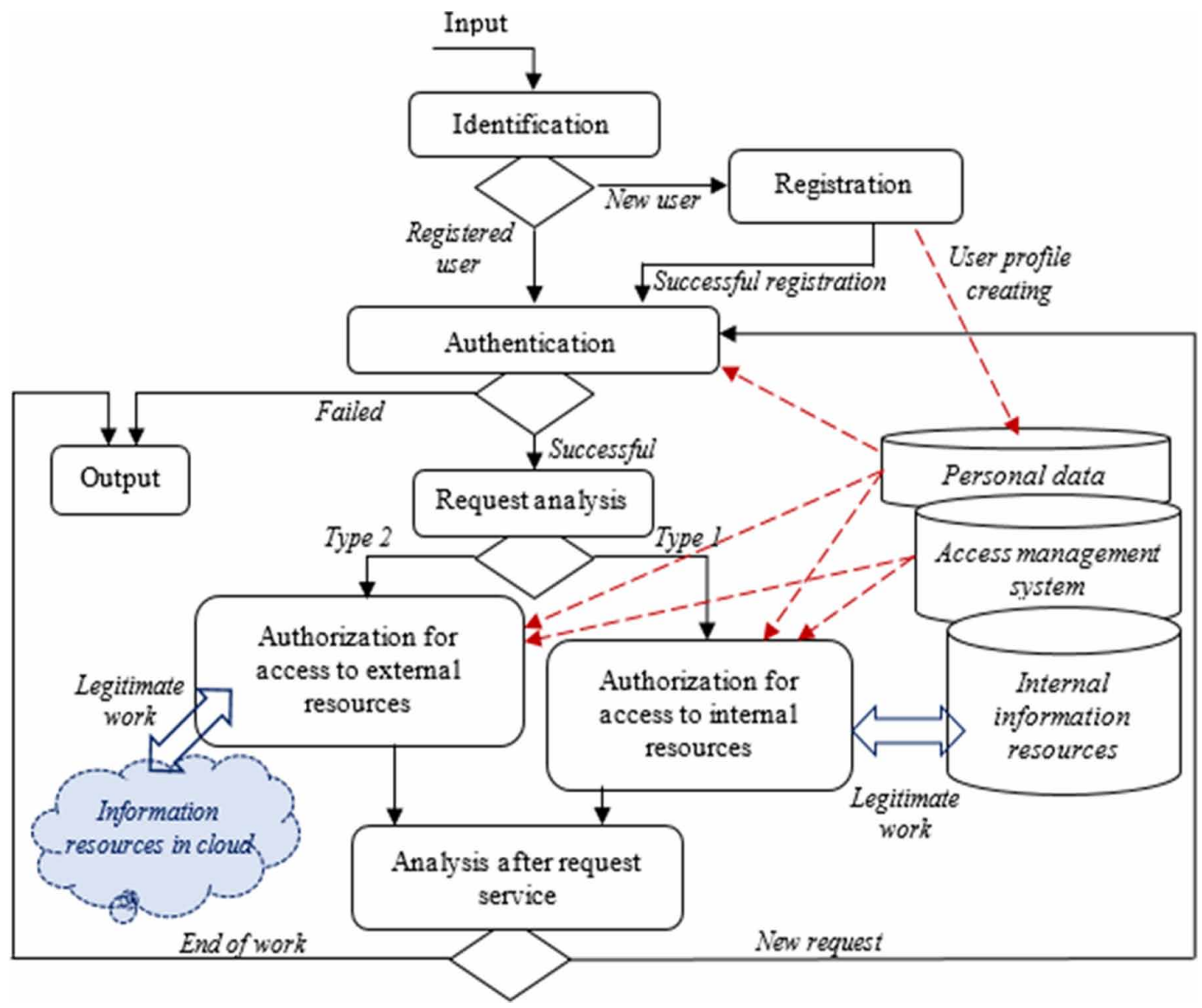

of data flow between elements in the model). It is permissible for a given source of data flow (requests for access in the case of HeLS) to be also a receiver of the final result or additional information (see "User" in the DFD chart).

DFD is a suitable tool for creating a visual sheme of the movement of data from between planned processes in the development (software and architecture) of a system. This preliminary model allows to decompose the general functionality in the form of separate relatively independent details (processes), activated in a certain sequence. The capabilities of DFD determine their wide applicability in various fields, including the design of processes to ensure reliable security and data protection. For example, in (Chong \& Diamantopoils, 2020) is presented a research for the integration of modern technologies in solving problems of Security of payment (SOP), and in the initial definition of the functional framework a DFD was developed to describe the overall process and formulate its main components.

\section{DETERMINISTIC MODEL INVESTIGATION}

The task of conceptual modeling is to make an appropriate presentation of data flows and their management in the implementation of processes in a system. This is especially important in the development of conventional and non-traditional process management systems in the global network, as the next phase is to confirm the correct organization by conducting a model investigation. A suitable environment for conducting such investigation is offered by the apparatus of Petri nets, and its 
Figure 5. Data Flow Diagram (DFD) described the main processes in the system

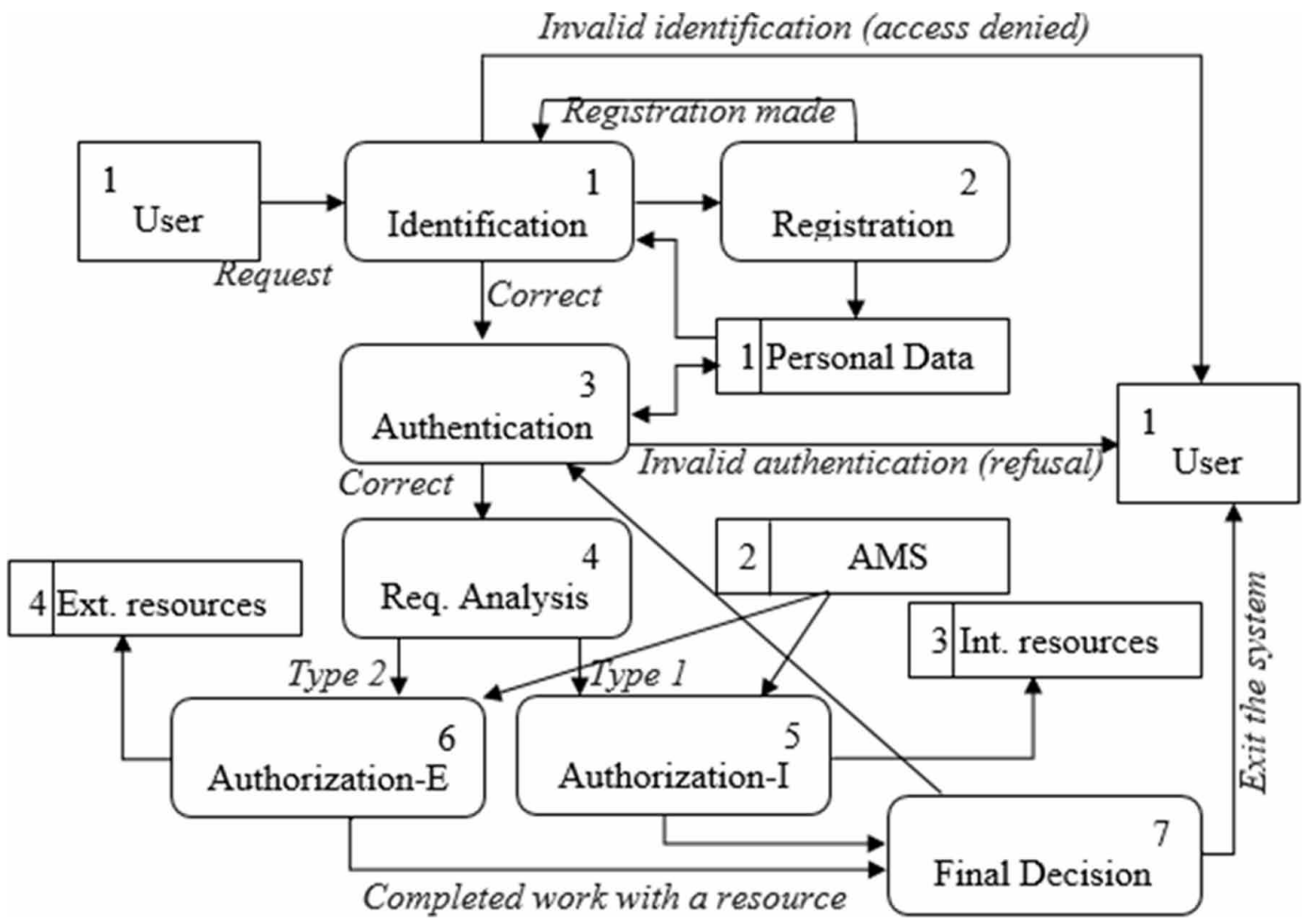

relevance has been confirmed by numerous publications. For example, in (Liu et al, 2020) an effective way is proposed to conduct a systematic modeling and analysis of the data flow using a Petri net, in which a modification of the classical variant is made by introducing read and write semantics used in modeling of control and data flows. This approach is used to conduct a deterministic investigation of the proposed processes organization in discussed system.

\section{Analytical Definition of PN-model}

The organization of analytical deterministic investigation is based on a PN-model defined as an ordered structure of four components $P N=\{T, P, I, O\}$, for $T C ̧ P=Æ$, which theoretical-set definition and graph presentation are presented below including in Figure 6.

Set of transitions $T=\left\{t_{i} / i=1 \div 8\right\}$ in the PN-model: $\mathrm{t}_{1}$ - procedure for identification of an incoming request; $t_{2}$ - procedure for identification of an incoming request; $t_{3}$ - authentication procedure for determining a legitimate user; $\mathrm{t}_{4}$ - exit from the system in case of illegitimate access or in case of refusal of further service; $t_{5}$ - analysis of a legitimate request for access to a resource; $\mathrm{t}_{6}$ - authorization procedure for verification of access rights to external resources stored in a global storage; $\mathrm{t}_{7}-$ authorization procedure for checking the access rights to internal resources stored in own memories; $\mathrm{t}_{8}$ - end of the service on the current request and analysis of the next action. Procedures $\mathrm{t}_{6}$ and $\mathrm{t}_{7}$ use DRMS.

Set of positions $P=\left\{p_{j} / j=1 \div 7\right\}$ in the PN-model: $p_{1}$ - presence of a request for access to the system (entrance from an external source to the font office); $\mathrm{p}_{2}$ - request from an unregistered source (user); $\mathrm{p}_{3}$ - request from a registered source (user); $\mathrm{p}_{4}$ - presence of a result after authentication determining the type of access (legitimate or illegitimate); $\mathrm{p}_{5}$ - analysed request with certain access to 


$$
\begin{aligned}
& \text { Input functions: } \\
& I\left(t_{1}\right)=\left\{p_{1}\right\} \\
& I\left(t_{2}\right)=\left\{p_{2}\right\} \\
& I\left(t_{3}\right)=\left\{p_{3}, p_{3}\right\} \\
& I\left(t_{4}\right)=\left\{p_{4}\right\} \\
& I\left(t_{5}\right)=\left\{p_{4}\right\} \\
& I\left(t_{6}\right)=I\left(t_{7}\right)=\left\{p_{5}, p_{6}\right\} \\
& I\left(t_{8}\right)=\left\{p_{7}\right\}
\end{aligned}
$$

Output functions:$$
O\left(t_{1}\right)=\left\{p_{2}, p_{3}\right\}
$$$$
O\left(t_{2}\right)=\left\{p_{3}\right\}
$$$$
O\left(t_{3}\right)=\left\{p_{4}\right\}
$$$$
O\left(t_{4}\right)=\left\{p_{1}\right\}
$$$$
O\left(t_{5}\right)=\left\{p_{5}, p_{6}\right\}
$$$$
O\left(t_{6}\right)=O\left(t_{7}\right)=\left\{p_{7}\right\}
$$$$
O\left(t_{8}\right)=\left\{p_{4}\right\}
$$

(a) Analytical definition - input $I(t)$ and output $O(t)$ functions

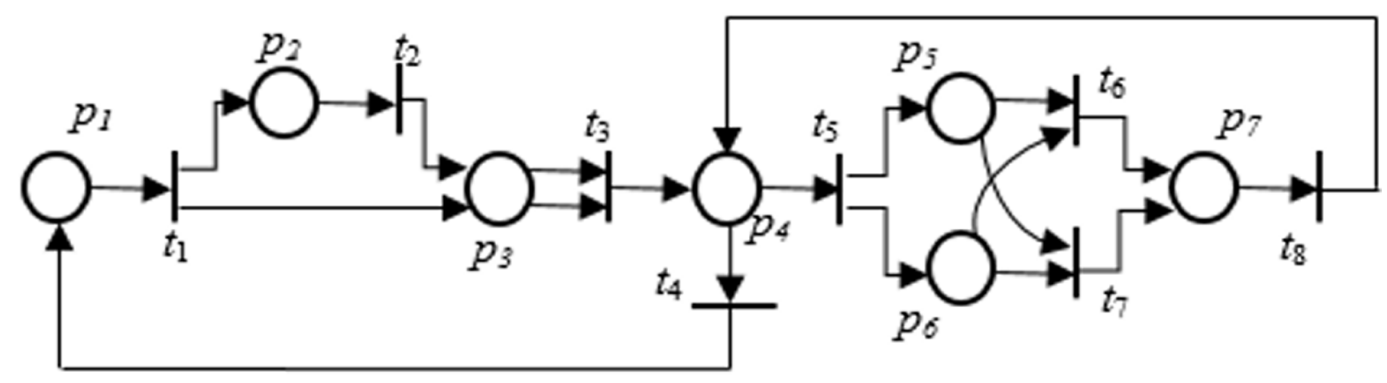

(b) Graph presentation of the PN-model

an external resource in a global storage; $\mathrm{p}_{6}$ - analysed request with certain access to internal resource in local storage; $\mathrm{p}_{7}$ - presence of a serviced request for determination of next action.

\section{Matrix Definition of PN-model}

As an extension, a matrix definition $P N=\left(P, T, \mathbf{D}^{-}, \mathbf{D}^{+}\right)$of the proposed $\mathrm{PN}$-model could be made by determining the two matrices: $\mathbf{D}^{-}$- input (reflecting the relationship between each transition and its input positions) and $\mathbf{D}^{+}$- output (reflects the relationship between each transition and its output positions), which are presented in the Figure 7:

In this case, each transition $t_{j}$ can be presented by $m$-dimensional vector $e[j](m=|T|)$.

$\mathbf{D}^{-}[j, i]=\eta\left(p_{i}, I\left(t_{j}\right)\right) ; \forall p_{i} \in P ; j=1 \div|T| ;$

$\mathbf{D}^{+}[j, i]=\eta\left(p_{i}, O\left(t_{j}\right)\right) ; \forall p_{i} \in P ; j=1 \div|T|$.

To determine whether a given transition $t_{\mathrm{j}+1}$ is allowed, it is analysed whether the condition $\mu_{j}^{3} e[j+1] . \mathrm{D}^{-}(j=0,1,2, \ldots)$ is fulfilled, where $e[j+1]$ is Boolean vector with only one " 1 " in position $(j+1)$ at $j=0,1,2, \ldots$ at each step, starting from the initial marking. In this case, with the given initial marking $\mu_{0}=(1,0,0,0,0,0,0)$ only transition $t_{1}$ is allowed, which is determined as follows:

$e[1]=(1,0,0,0,0,0,0)$ è $e[1] \cdot \mathrm{D}^{-}=(1,0,0,0,0,0,0) \cdot \mathrm{D}^{-}=(1,0,0,0,0,0,0) \mathrm{P}$ condition $\mu_{0}{ }^{3} \mathrm{e}[1] \cdot \mathrm{D}^{-}$is fulfilled and therefore the transition $t_{1}$ is allowed at marking $\mu_{0}$.

The situations for the next steps are similarly determined: 
$\mathbf{D}^{-}[j, i]=\eta\left(p_{i}, I\left(t_{j}\right)\right) ; \forall p_{i} \in P ; j=1 \div|T| ;$

$\mathbf{D}^{+}[j, i]=\eta\left(p_{i}, O\left(t_{j}\right)\right) ; \forall p_{i} \in P ; j=1 \div|T|$.

\begin{tabular}{|c|c|c|c|c|c|c|c|}
\hline $\mathbf{D}^{-}$ & $\mathrm{p}_{1}$ & $\mathrm{p}_{2}$ & $\mathrm{p}_{3}$ & $\mathrm{p}_{4}$ & $\mathrm{p}_{5}$ & $\mathrm{p}_{6}$ & $\mathrm{p}_{7}$ \\
\hline $\mathrm{t}_{1}$ & 1 & 0 & 0 & 0 & 0 & 0 & 0 \\
\hline $\mathrm{t}_{2}$ & 0 & 1 & 0 & 0 & 0 & 0 & 0 \\
\hline $\mathrm{t}_{3}$ & 0 & 0 & 2 & 0 & 0 & 0 & 0 \\
\hline $\mathrm{t}_{4}$ & 0 & 0 & 0 & 1 & 0 & 0 & 0 \\
\hline $\mathrm{t}_{5}$ & 0 & 0 & 0 & 1 & 0 & 0 & 0 \\
\hline $\mathrm{t}_{6}$ & 0 & 0 & 0 & 0 & 1 & 1 & 0 \\
\hline $\mathrm{t}_{7}$ & 0 & 0 & 0 & 0 & 1 & 1 & 0 \\
\hline $\mathrm{t}_{8}$ & 0 & 0 & 0 & 0 & 0 & 0 & 1 \\
\hline
\end{tabular}

\begin{tabular}{|c|c|c|c|c|c|c|c|}
\hline $\mathbf{D}^{+}$ & $\mathrm{p}_{1}$ & $\mathrm{p}_{2}$ & $\mathrm{p}_{3}$ & $\mathrm{p}_{4}$ & $\mathrm{p}_{5}$ & $\mathrm{p}_{6}$ & $\mathrm{p}_{7}$ \\
\hline $\mathrm{t}_{1}$ & 0 & 1 & 1 & 0 & 0 & 0 & 0 \\
\hline $\mathrm{t}_{2}$ & 0 & 0 & 1 & 0 & 0 & 0 & 0 \\
\hline $\mathrm{t}_{3}$ & 0 & 0 & 0 & 1 & 0 & 0 & 0 \\
\hline $\mathrm{t}_{4}$ & 1 & 0 & 0 & 0 & 0 & 0 & 0 \\
\hline $\mathrm{t}_{5}$ & 0 & 0 & 0 & 0 & 1 & 1 & 0 \\
\hline $\mathrm{t}_{6}$ & 0 & 0 & 0 & 0 & 0 & 0 & 1 \\
\hline $\mathrm{t}_{7}$ & 0 & 0 & 0 & 0 & 0 & 0 & 1 \\
\hline $\mathrm{t}_{8}$ & 0 & 0 & 0 & 1 & 0 & 0 & 0 \\
\hline
\end{tabular}

$e[2]=(0,1,0,0,0,0,0)$ è $e[2] \cdot \mathrm{D}^{-}=(0,1,0,0,0,0,0) \cdot \mathrm{D}^{-}=(0,1,0,0,0,0,0) \mathrm{P}$ condition $\mu_{1}{ }^{3} \mathrm{e}[2] \cdot \mathrm{D}^{-}$is fulfilled and allowed transition at this marking $\mu_{1}$ is $\mathrm{t}_{2}$.

$e[3]=(0,0,1,0,0,0,0)$ è $e[3] \cdot \mathrm{D}^{-}=(0,0,1,0,0,0,0) \cdot \mathrm{D}^{-}=(0,0,2,0,0,0,0) \mathrm{P}$ condition $\mu_{2}{ }^{3} \mathrm{e}[3] \cdot \mathrm{D}^{-}$is fulfilled and $t_{3}$ is allowed at $\mu_{2}$.

To determine the next marking $\mu^{*}$ when activating the transition $t_{\mathrm{j}}$, the equation $\mu^{*}=e[j] . D$ is applied, where the incidental matrix $D=D^{+}-D^{-}$is the following:

In this case, at the initial marking $\mu_{0}=(1,0,0,0,0,0,0)$ the next marking is determined on the base of the calculation

$\mu_{1}=\mu_{0}+e\left[t_{1}\right] \cdot D=\mu_{0}+e[1] \cdot D=(1,0,0,0,0,0,0)+(1,0,0,0,0,0,0) \cdot D=$

$=(1,0,0,0,0,0,0)+(-1,+1,0,0,0,0,0)=(0,+1,+1,0,0,0,0) \mathrm{P} \mu_{1}=(0,1,1,0,0,0,0)$

\section{Evaluation of the PN-model}

The evolution of the PN-model as a sequence of the determined markings when activating the allowed transitions in each step, starting from the initial marking $\mu_{0}=(1,0,0,0,0,0,0)$, is presented in Figure 8. A generalized structure of the tree of reachability is given at the bottom of the figure.

It is known that Petri nets are an asynchronous apparatus, and in evolution in each step of the deterministic implementation only one of all allowed transitions is activated, which creates branches in the tree of reachability. In the specific case, when performing the PN-model, possible branches in steps $4\left(\mu_{3}\right)$ and $5\left(\mu_{4}\right)$ are observed, as well as two cyclic sequences $\mu_{0}{ }^{\circledR} \mu_{1}{ }^{\circledR} \mu_{2}{ }^{\circledR} \mu_{3}{ }^{\circledR} \mu_{0}$ and $\mu_{3}{ }^{\circledR} \mu_{4}{ }^{\circledR} \mu_{5}{ }^{\circledR} \mu_{3}$. The evolution of the model as a sequence of steps indicating the allowed transitions and their possible implementation is given in Table 1 .

The investigation of the model was performed by analysing the tree of reachability and the main properties for the Petri net. The results can be summarized as follows:

- Reachability - the model allows cyclic ignition (activation) of the transitions with repeatability of the separate phases. 
Figure 7b. Calculation determination

\begin{tabular}{|c|c|c|c|c|c|c|c|}
\hline $\boldsymbol{D}$ & $\mathrm{p}_{1}$ & $\mathrm{p}_{2}$ & $\mathrm{p}_{3}$ & $\mathrm{p}_{4}$ & $\mathrm{p}_{5}$ & $\mathrm{p}_{6}$ & $\mathrm{p}_{7}$ \\
\hline $\mathrm{t}_{1}$ & -1 & +1 & +1 & 0 & 0 & 0 & 0 \\
\hline $\mathrm{t}_{2}$ & 0 & -1 & +1 & 0 & 0 & 0 & 0 \\
\hline $\mathbf{t}_{3}$ & 0 & 0 & -2 & +1 & 0 & 0 & 0 \\
\hline $\mathbf{t}_{4}$ & +1 & 0 & 0 & -1 & 0 & 0 & 0 \\
\hline $\mathbf{t}_{5}$ & 0 & 0 & 0 & -1 & +1 & +1 & 0 \\
\hline $\mathbf{t}_{6}$ & 0 & 0 & 0 & 0 & -1 & -1 & +1 \\
\hline $\mathbf{t}_{7}$ & 0 & 0 & 0 & 0 & -1 & -1 & +1 \\
\hline $\mathbf{t}_{8}$ & 0 & 0 & 0 & +1 & 0 & 0 & -1 \\
\hline
\end{tabular}

Figure 8. Tree of reachability of the PN-model

$$
\begin{aligned}
& \mu_{0}=(1,0,0,0,0,0,0) \stackrel{t 1}{\longrightarrow} \mu_{1}=(0,1,1,0,0,0,0) \stackrel{t 2}{\longrightarrow} \mu_{2}=(0,0,2,0,0,0,0) \\
& \mu_{2} \stackrel{t^{3}}{\longrightarrow} \mu_{3}=(0,0,0,1,0,0,0) \\
& \mu_{3} \stackrel{t 4}{\longrightarrow}(1,0,0,0,0,0,0) \equiv \mu_{0} \\
& \mu_{3} \stackrel{t^{5}}{\longrightarrow} \mu_{4}(0,0,0,0,1,1,0) \\
& \mu_{4} \stackrel{t 6}{\longrightarrow} \mu_{51}(0,0,0,0,0,0,1) \stackrel{t_{8}}{\longrightarrow}(0,0,0,1,0,0,0) \equiv \mu_{3} \\
& \mu_{3} \stackrel{t_{7}}{\longrightarrow} \mu_{52}(0,0,0,0,0,0,1) \stackrel{t_{8}}{\longrightarrow}(0,0,0,1,0,0,0) \equiv \mu_{3}
\end{aligned}
$$

- Liveness - the property is executed because from the set initial marking in evolution there is at least one allowed transition for each step of the execution (see Table 1);

- Blocking - there are no locks, which makes the model active, as in single-user (monopoly) access to information resources no conflict situations arise;

- Boundness - at the given initial marking the model is 2-limited, because the number of marks in each position does not exceed the number 2 for each marking, achievable by $\mu 0$, i.e. $\Sigma \mu\left(p_{\mathrm{i}}\right) £ 2$ (see Table 1);

- Safety - it can be assumed that the model is safe, despite the fact that there are 2-safe (2-limited) positions, because for the transitions associated with these positions, there are two input and two output arcs. 
Table 1. Evolution of the PN-model

\begin{tabular}{|c|c|c|c|}
\hline mcurrent & Allowed transition & Activated transition & mnew \\
\hline $\mathrm{m} 0$ & $\mathrm{t} 1$ & $\mathrm{t} 1$ & $\mathrm{~m} 1=(1,0,0,0,0,0,0)$ \\
\hline $\mathrm{m} 1$ & $\mathrm{t} 2$ & $\mathrm{t} 2$ & $\mathrm{~m} 2=(0,1,1,0,0,0,0)$ \\
\hline $\mathrm{m} 2$ & $\mathrm{t} 3$ & $\mathrm{t} 3$ & $\mathrm{~m} 3=(0,0,2,0,0,0,0)$ \\
\hline $\mathrm{m} 3$ & $\mathrm{t} 4 \& \mathrm{t} 5$ & $\mathrm{t} 4$ & $\mathrm{~m} 0=(0,0,0,1,0,0,0)$ \\
\hline $\mathrm{m} 4$ & $\mathrm{t} 6 \& \mathrm{t} 7$ & $\mathrm{t} 6$ & $\mathrm{~m} 4=(0,0,0,0,1,1,0)$ \\
\hline $\mathrm{m} 51$ & $\mathrm{t} 8$ & $\mathrm{t} 7$ & $\mathrm{~m} 51=(0,0,0,0,0,0,1)$ \\
\hline $\mathrm{m} 52$ & $\mathrm{t} 8$ & $\mathrm{t} 8$ & $\mathrm{~m} 52=(0,0,0,0,0,0,1)$ \\
\hline
\end{tabular}

\section{CONCLUSION AND DISCUSSION}

The development of information technologies, globalization and informatization of society, increasing the speed of network and mobile communications, the use of smartphones, etc. lead to the transformation of e-learning technologies, making it more efficient and allowing the creation of new innovative forms of e-learning: ü Micro-learning - development of micro-educational activities through small steps in digital media environments, forming separate educational sessions in the educational process; $\ddot{u}$ Gamification - the use of games and game mechanisms in the development of non-game content used to solve problems; ü Personalized learning - development of pedagogical, teaching and educational environments designed for specific learners by adapting to the desired educational style of the learners and the level of intelligence.

The article discusses another contemporary possibility - the application of technologies for Cloud $\&$ Social Computing as a prerequisite for the development of e-learning. In this case, it is proposed to use the cloud to increase the efficiency of information and data flow management through the use of external data storages and additional cloud services in the organization of resources and processes. Additional opportunities are provided through the use of social networks for active communication between students and teachers, which will allow adequate sharing of knowledge and resources. For the realization of the proposed activities in the article the architecture of the training environment is presented and an analysis of the organizational processes is carried out, paying attention to the security in access to resources and data protection

To fulfill the set goal in the proposed architectural concept of a heterogeneous learning environment are implemented opportunities for resource management and processes with regulated access and verification of the authorization. Several levels of regulation are defined - initial identification, authentication and authorization, as for checking the correctness of processes and application of procedures a study was conducted using the possibilities of mathematical formalization, conceptual modeling, structural design, Data Flow Diagram and PN-apparatus for deterministic modelling. The analysis of the experimental results and properties of the analytical PN-model confirms the adequacy of the processes and the absence of "bottlenecks" that would lead to blockages and denial of service.

As a next task for future research, an extension of the model investigation can be made by applying stochastic PN and probabilistic analysis of evolution through a Markov chain. This would make it possible to determine the conditions for reaching steady state by calculating numerical values for observable factors when varying the values for the controllable factors. 


\section{REFERENCES}

Cai, F., He, J., Ali Zardari, Z., \& Han, S. (2020). Distributed management of permission for access control model. Journal of Intelligent \& Fuzzy Systems, 38(2), 1539-1548. doi:10.3233/JIFS-179517

Chatterjee, S. (2021). Antecedence of attitude toward IoT usage: A proposed unified model for IT Professionals and its validation. International Journal of Human Capital and Information Technology Professionals, 12(2), 13-34. doi:10.4018/IJHCITP.2021040102

Cheryshov, A.B., Choporov, O.N., Preobrazhenskiy, P.A., \& Kravets, O.Ja. (2020). The development of optimization model and algorithm for support of resources management in organizational system. International Journal on Information Technologies and Security, 12(2), 25-36.

Chong, H.-Y., \& Diamantopoulos, A. (2020). Integrating advanced technologies to uphold security of payment: Data flow diagram. Automation in Construction, 114(June), 103158. Advance online publication. doi:10.1016/j. autcon.2020.103158

Christie, M. A., Bhandar, A., Nakandala, S., Marru, S., Abeysinghe, E., Pamidighantam, S., \& Pierce, M. E. (2020). Managing authentication and authorization in distributed science gateway middleware. Future Generation Computer Systems, 111(October), 780-785. doi:10.1016/j.future.2019.07.018

Digalovski, M., \& Rafajlovski, G. (2020). Distribution transformer mathematical model for power losses minimization. International Journal on Information Technologies and Security, 12(2), 57-68.

Ding, C., Niu, Z., Li, B., Hog, D., Zhang, Z., \& Yu, M. (2020). Analytical modeling and thermal performance analysis of a flat plate latent heat storage unit. Applied Thermal Engineering, 179(October), 115722. doi:10.1016/j. applthermaleng.2020.115722

Egoeze, F., Misra, S., Maskeliūnas, R., \& Damaševičius, R. (2018). Impact of ICT on universities administration services and management of stucents' records: ICT in university administration. International Journal of Human Capital and Information Technology Professionals, 9(2), 1-15. doi:10.4018/JJHCITP.2018040101

Glet, M., \& Kaczyński, K. (2020). Secret sharing scheme for creating multiple secure storage dimensions for mobile applications. International Journal on Information Technologies and Security, 12(4), 83-102.

Hiran, K. K. (2021). Investigating factors influencing the adoption of IT cloud computing platforms in higher education: Case of Sub-Saharan Africa with IT professionals. International Journal of Human Capital and Information Technology Professionals, 12(3), 21-36. doi:10.4018/IJHCITP.2021070102

Iakushkin, O. (2020). Service-oriented Petri net model. In Computational Science and Its Applications - ICCSA 2020. In Lecture Notes in Computer Science (Vol. 12254). Springer. doi:10.1007/978-3-030-58817-5_5

Kai, H., Al-Ahmari, A., Li, Z., \& Davidrajuh, R. (2020). Single controller-based colored Petri nets for deadlock control in automated manufacturing systems. Processes (Basel, Switzerland), 8(1), 21. Advance online publication. doi: $10.3390 / p r 80100021$

Kanakaris, V., Lampropoulos, G., \& Siakas, K. (2019). A survey and a case-study regarding media security and privacy on Greek future IT professionals. International Journal of Human Capital and Information Technology Professionals, 10(1), 22-37. doi:10.4018/IJHCITP.2019010102

Khouja, M., Rodriguez, I. B., Ben Halima, Y., \& Moalla, S. (2018). IT governance in higher education institutions: A systematic literature review. International Journal of Human Capital and Information Technology Professionals, 9(2), 52-67. doi:10.4018/IJHCITP.2018040104

Li, Q., \& Chen, Y. L. (2009). Data Flow Diagram. In Modeling and Analysis of Enterprise and Information Systems. Springer. doi:10.1007/978-3-540-89556-5_4

Liu, C., Zeng, Q., Duan, H., Wang, L., Tan, J., Ren, C., \& Yu, W. (2020). Petri Net Based Data-Flow Error Detection and Correction Strategy for Business Processes. IEEE Access: Practical Innovations, Open Solutions, 8(Feb), 43265-43276. doi:10.1109/ACCESS.2020.2976124

Liu, F., Heiner, M., \& Gilbert, D. (2020). Fuzzy Petri nets for modelling of uncertain biological systems. Briefings in Bioinformatics, 21(1), 198-210. PMID:30590430 
Monev, V. (2020). Measuring the Optimal Information Security Complexity for Blockchain Operations. 2020 International Conference on Information Technologies (InfoTech-2020 Proceedings), 57-60.

Oleinikova, S.A., \& Kravets, O.J., \& Atlasov, I.V. (2019). The heuristic algorithm for solving the optimization problem of the start time of jobs determining from the generalized resource criterion point of view. International Journal on Information Technologies and Security, 11(3), 69-78.

Romansky, R., \& Noninska, I. (2016). Architecture of combined e-learning environment and investigation of secure access and privacy protection. International Journal of Human Capital and Information Technology Professionals, 7(3), 89-106. doi:10.4018/IJHCITP.2016070107

Romansky, R., \& Noninska, I. (2019). Technological Organization of the Access Management to Information Resources in a Combined e-Learning Environment. International Journal on Information Technologies and Security, 11(4), 51-62.

Romansky, R., \& Noninska, I. (2020a). Challenges of the digital age for privacy and personal data protection. Mathematical Biosciences and Engineering, 17(5), 5288-5303. doi:10.3934/mbe.2020286 PMID:33120553

Romansky, R., \& Noninska, I. (2020b). Business virtual system in the context of e-governance: Investigation of secure access to information resources. Journal of Public Affairs, 20(3), 2072. Advance online publication. doi:10.1002/pa.2072

Taleb-Berrouane, M., Khan, F., \& Amyotte, P. (2020). Bayesian stochastic Petri nets (BSPN) - A new modelling tool for dynamic safety and reliability analysis. Reliability Engineering \& System Safety, 193, 106587. doi:10.1016/j.ress.2019.106587

Tay, Y. C. (2020). A review of analytical performance modeling and its role in computer engineering and science. Cornell University. https://arxiv.org/abs/2005.13144

Torres, N.V., \& Santos, G. (2015). The (mathematical) modeling process in biosciences. Frontiers in Genetics (System Biology), 6(354). .10.3389/fgene.2015.00354 